\title{
Traços de Linguagem Revista de Estudos Linguísticos
}




\section{UNEMAT \\ Universidade do Estado de Mato Grosso}

Reitora

Ana Maria Di Renzo

Vice-Reitor

Ariel Lopes Torres

Pró-Reitor de Pesquisa e Pós-Graduação

Rodrigo Bruno Zanin

Apoio:

PROEC

Pró-Reitoria de Extensão e Cultura
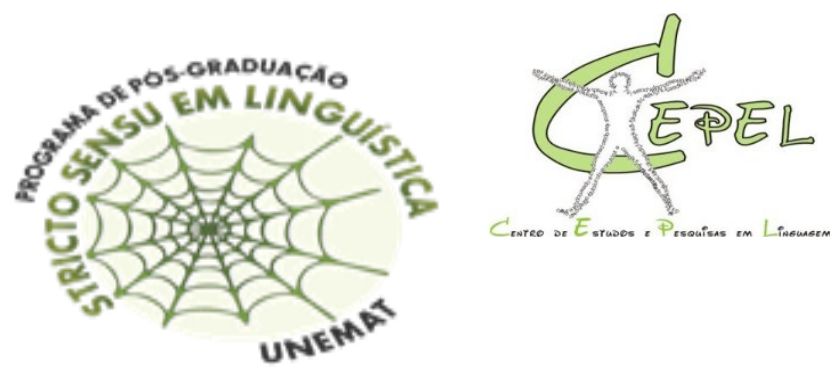


\section{UNEMAT \\ Universidade do Estado de Mato Grosso}

\section{Traços de Linguagem Revista de Estudos Linguísticos}

Traços de Linguagem, Cáceres, v. 2, n. 2, p. 1-82, 2018.

ISSN - 2594-9063 


\title{
Expediente
}

Traços de Linguagem - Revista de Estudos Linguísticos

UNEMAT - Universidade do Estado de Mato Grosso

Pró-Reitoria de Extensão e Cultura

Grupo de Pesquisa Variação e Invariantes na Linguagem

Programa de Pós-Graduação em Linguística - Cidade Universitária - Av. Santos Dumont s/n - Bloco II - Centro de Pesquisa e Pós-Graduação em Linguagem - Bairro DNER - CEP: 78.200-000 - Cáceres/MT.

Editor Responsável
Albano Dalla Pria

Editoria Executiva
Albano Dalla Pria
Jocineide Macedo Karim
Marcos Luiz Cumpri
Silvia Regina Nunes
Taisir mahmudo Karim
Suporte Técnico
Tikao Tsukamoto Júnior

\author{
Revisão Geral \\ Marcos Luiz Cumpri \\ Diagramação \\ Fátima Graziele de Souza \\ Albano Dalla Pria \\ Capa \\ Marta de Paula Vieira de Paula Vieira \\ Joseléia Graciano
}

Roberto Tikao Tsukamoto Júnior

\section{Conselho Editorial}

Adriana Zavaglia (USP), Angel Corbera Mori (UNICAMP), Carlos Alexandre Victorio Gonçalves (UFRJ), Dircel Aparecida Kailer (UEL), Eduardo Junqueira Guimarães (UNICAMP), Eni Puccinelli Orlandi (UNIVAS), Freda Indurski (UFRGS), Letícia Marcondes Rezende (UNESP), Luiz Francisco Dias (UFMG), Márcia Cristina Romero

Lopes (UNIFESP), Maria Auxiliadora Ferreira Lima (UFPI), Milenne Biasotto (UFGD), Rosane de Andrade Berlinck (UNESP), Sebastião Carlos Leite Gonçalves (UNESP).

\section{Revisores}

Albano Dalla Pria (UNEMAT), Gislaine Aparecida de Carvalho (UNEMAT), Jocineide Macedo Karim (UNEMAT), Marcos Luiz Cumpri (UNEMAT/CAPES), Elaine Aguiar Florêncio (UNEMAT/PPGL), Fátima Graziele de Souza (UNEMAT/PPGL), Weverton Ortiz Fernandes (UNEMAT/PPGL), Tiego Vaz Sardinha (UNEMAT/PPGL), Marta de Paula Vieira de Paula Vieira (UNEMAT/PPGL).

\section{Publicação semestral.}

ISSN: $2594-9063$

Traços de Linguagem - Revista de Estudos Linguísticos.

Eric Fernández Hernández, Gislaine Aparecida de Carvalho, Marcos Luiz Cumpri (Orgs.)

Cáceres, MT: Universidade do Estado de Mato Grosso, 2018. 82 p.

1. Linguística 2. Linguagem 3 . Línguas

Semestral (Ref.: jul-dez, 2018.) Vol. 2, n. 2. (2018)-

CDU: 81

Ficha catalográfica elaborada pela equipe do Centro de Pesquisas em Linguagem - CEPEL - Cáceres.

Índice para Catálogos Sistemáticos

1. Linguística -81 